Mathematical Modelling and Analysis

Volume 16 Number 1, March 2011, 72-81

Doi:10.3846/13926292.2011.560616

(C) Vilnius Gediminas Technical University, 2011
www.tandf.co.uk/journals/TMMA

Publisher: Taylor\&Francis and VGTU

Online ISSN: 1648-3510

Print ISSN: 1392-6292

\title{
Questions around the Nontrivial Zeros of the Riemann Zeta-Function. Computations and Classifications*
}

\author{
R. Garunkštis ${ }^{a}$ and J. Steuding ${ }^{b}$ \\ ${ }^{a}$ Vilnius University \\ Naugarduko 24, LT-03225 Vilnius, Lithuania \\ ${ }^{b}$ Würzburg University \\ Am Hubland, 97218 Würzburg, Germany \\ E-mail(corresp.): ramunas.garunkstis@mif .vu.1t \\ E-mail: steuding@mathematik. uni-wuerzburg.de
}

Received August 7, 2010; revised Januar 9, 2011; published online March 1, 2011

\begin{abstract}
We study the sequence of nontrivial zeros of the Riemann zeta-function with respect to sequences of zeros of other related functions, namely, the Hurwitz zeta-function and the derivative of Riemann's zeta-function. Finally, we investigate connections of the nontrivial zeros with the periodic zeta-function. On the basis of computation we derive several classifications of the nontrivial zeros of the Riemann zeta-function and state problems which might be of interest for a better understanding of the distribution of those zeros.
\end{abstract}

Keywords: Riemann zeta-function, Hurwitz zeta-function, periodic zeta-function, nontrivial zeros.

AMS Subject Classification: 11M26; 11M35.

\section{Introduction}

Let $s=\sigma+i t$ denote a complex variable. For $\sigma>1$, the Riemann zeta-function is given by

$$
\zeta(s)=\sum_{n=1}^{\infty} \frac{1}{n^{s}} .
$$

This function can be analytically continued to the whole complex plane except for a simple pole at $s=1$ with residue 1 . Trivial zeros of $\zeta(s)$ are located at the negative even integers. The remaining, so- called nontrivial zeros lie in the critical strip $0<\sigma<1$. In this paper we discuss several relations of those nontrivial zeros with respect to zeros of related functions. In the

\footnotetext{
* The first author is supported by grant No MIP-94 from the Research Council of Lithuania.
} 
following sections we distinguish between 'stable' and 'unstable' zeros based on the trajectories of zeros of the Hurwitz zeta-function, notions are introduced in [2]. In Section 3 we investigate how the nontrivial zeros are related via trajectories with those of $\zeta^{\prime}(s)$. Section 4 is devoted to a classification of zeros by properties of the periodic zeta-function.

\section{Stable and Unstable Zeros}

For $\sigma>1$, the Hurwitz zeta-function is given by

$$
\zeta(s, \alpha)=\sum_{n=0}^{\infty} \frac{1}{(n+\alpha)^{s}},
$$

where $\alpha$ is a parameter from the interval $(0,1]$. The Hurwitz zeta-function can be continued analytically to the whole complex plane except for a simple pole at $s=1$ with residue 1 . For $\alpha=1$ the Hurwitz zeta-function becomes the Riemann zeta-function $\zeta(s)=\zeta(s, 1)$. As a matter of fact, we have further

$$
\zeta(s, 1 / 2)=\left(2^{s}-1\right) \zeta(s)
$$

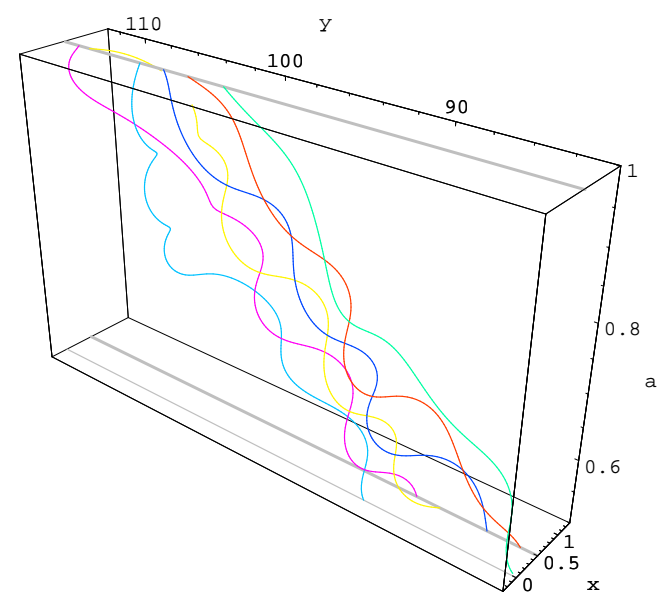

Figure 1. Trajectories of several zeros of $\zeta(s, \alpha), 0.5 \leq \alpha \leq 1$; the 30-th zero of $\zeta(s)=\zeta(s, 1)$ is plotted in green, the 35 -th zero in pink.

Consider Figure 1, where several zeros trajectories of $\zeta(s, \alpha)$ are shown, as $\alpha$ varies in the range $0.5 \leq \alpha \leq 1$. For example, it shows that the trajectories which start at the 30-th and the 33-rd zero of $\zeta(s)=\zeta(s, 1)$ end at zeros of $\zeta(s, 1 / 2)$ on the line $\sigma=1$. We recall some notions introduced in [2]. We call a zero $\rho$ of $\zeta(s)$ stable if its trajectory ends on the critical line as $\alpha \rightarrow 1 / 2$; otherwise the zero is called unstable. Denoting the zeros of $\zeta(s)$ with positive ordinate by $\rho_{n}=\beta_{n}+i \gamma_{n}$ (in ascending order), we find among the first 501 zeros the following unstable zeros, indicated by their index $n$ : 
$1,3,6,9,13,17,21,26,30,33,40,44,50,54,61,67,70,78,79$, $90,93,101,109,112,117,124,134,139,147,149,153,165,167$, $175,186,189,197,201,214,218,219,234,235,240,253,255,266$, $270,275,282,288,299,300,313,317,334,342,344,355,359,370$, 371, 384, 387, 394, 409, 418, 422, 431, 434, 444, 450, 459, 465, 477, 489, 493, 500, 501 .

Note that in [2] the list of unstable zeros is correct only up to the 288th zero. Here we present the corrected list of unstable zeros. We are grateful to Jonathan Sondow whose notes helped us to find inaccuracies in the previous list of unstable zeros.

Figure 1 suggests that the zeros for $\alpha=1$ should migrate to zeros of smaller imaginary part at $\alpha=1 / 2$. The number of nontrivial zeros of $\zeta(s)$ and $\zeta(s, 1 / 2)$ up to $T$ is asymptotically equal to

$$
\frac{T}{2 \pi} \log \frac{T}{2 \pi e}+O(\log T) \quad \text { and } \quad \frac{T}{2 \pi} \log \frac{T}{2 \pi e}+\frac{T}{2 \pi} \log 2+O(\log T),
$$

respectively (see [3]), and $\frac{T}{2 \pi} \log 2+O(1)$ many of these zeros of $\zeta(s, 1 / 2)$ lie on the line $\sigma=0$, namely exactly those of the factor $2^{s}-1$ in (2.1). Therefore, we may expect that the number of unstable zeros up to $T$ is asymptotically equal to

$$
T \frac{\log 2}{2 \pi}\left(1-\frac{\log 2}{\log \frac{T}{2 \pi e}}\right) .
$$

On average, we conjecture that about $\frac{1}{\log 2} \log \frac{T}{2 \pi e}$ stable zeros lie in between two consecutive unstable zeros with imaginary part approximately equal to $T$. We do not expect many pairs of consecutive unstable zeros. Among the first 501 zeros (with positive real part) we have found only six pairs of such unstable twins:

$78,79,218,219,234,235,299,300,370,371$, and 500, 501.

The computations in this section are based on numerical solutions of the differential equation

$$
\frac{\partial z_{0}(\alpha)}{\partial \alpha}=-\frac{\partial \zeta(z, \alpha)}{\partial \alpha} / \frac{\partial \zeta(z, \alpha)}{\partial z},
$$

where $z=z_{0}(\alpha), \zeta\left(z_{0}(\alpha), \alpha\right)=0$. For the initial condition the zeros of $\zeta(s, 1)$ have been used, resp. zeros of $\zeta(s, 1 / 2)$ to check the calculations.

\section{Multiple $a$-Values and Zeros}

Let $\rho^{\prime}$ be a zero of the derivative of the Riemann zeta-function $\zeta^{\prime}(s)$ and let $a=\zeta\left(\rho^{\prime}\right)$. Then $\rho^{\prime}$ is a multiple $a$-value of $\zeta(s)$. We consider a trajectory $s(x)$, defined by the equation

$$
\zeta(s(x))-a x=0,
$$

where $x \in[0,1], s(1)=\rho^{\prime}$ and $s(0)$ must be a zero of $\zeta(s)$. Concerning trajectory $s(x)$ we have the following proposition. 
Proposition 1. Let $a \neq 0$ be a complex number. For $x=x_{0}$ let $s_{0}$ be a zero of multiplicity $m$ of

$$
f_{x}(s):=\zeta(s)-a x .
$$

Let $D\left(s_{0}\right)$ be a closed disc with center at $s_{0}$ in which the function $f_{x_{0}}(s)$ has no other zeros except $s_{0}$. Then there exists some $\delta>0$ such that any function $f_{x}(s)$ with $x \in\left(x_{0}-\delta, x_{0}+\delta\right)$ has exactly $m$ zeros (counted with multiplicities) in the disc $D\left(s_{0}\right)$. Moreover, if $m=1$, then there is a function $s(x)$, defined for $x \in\left(x_{0}-\delta, x_{0}+\delta\right)$, which is continuous at $x=x_{0}$ and satisfies the relation (3.1).

Proof. For any $s \neq 1$ the function $f_{x}(s)$ is continuous in $x$. Hence, the proposition follows from Rouché's theorem.

Since $\rho^{\prime}$ is a multiple $a$-value of $\zeta(s)$, we expect at least two solutions $s_{1}(x)$ and $s_{2}(x)$ of the equation $(3.1)$, with $s_{1}(1)=s_{2}(1)=\rho^{\prime}$ and $s_{1}(0) \neq s_{2}(0)$. To find a trajectory $s(x)$ we solve numerically the differential equation

$$
\frac{\partial \zeta(s(x))}{\partial s(x)} \frac{\partial s(x)}{\partial x}-a=0
$$

with initial condition $s(0)=\rho$, where $\rho$ is an appropriate $\zeta(s)$ zero, which we find experimentally. Note, that we cannot start with the initial condition $s(1)=\rho^{\prime}$, as $\rho^{\prime}$ is a multiple value of $\zeta(s)$.

We enumerate zeros of $\zeta^{\prime}(s)$ in $-1 \leq \sigma \leq 3, t>0$, in ascending order with respect to their imaginary parts: $\rho_{1}^{\prime}, \rho_{2}^{\prime}, \ldots$ Similarly we enumerate nontrivial zeros of $\zeta(s): \rho_{1}, \rho_{2}, \ldots$ as we did in the previous section.

Now we consider equation $(3.1)$ for $a=\zeta\left(\rho_{1}^{\prime}\right)=0.92 \ldots+i 0.03 \ldots$, where $\rho_{1}^{\prime}=2.46 \ldots+i 23.29 \ldots$ is the first zero of the derivative of the Riemann zetafunction. We look for two solutions of the differential equation (3.2), each of which starts at some zero of the Riemann zeta-function and ends at $\rho_{1}^{\prime}$. After some experiments we find solutions $s_{1}(x)$ and $s_{2}(x)$, where $x \in[0,1]$, $s_{1}(0)=\rho_{2}, s_{2}(0)=\rho_{3}$ and $s_{1}(1)=s_{2}(1)=\rho_{1}^{\prime}$ (see Figure 2). Based on this, we introduce the notation $(2,3)_{1}$ and say that zeros $\rho_{2}$ and $\rho_{3}$ of $\zeta(s)$ are related by the zero $\rho_{1}^{\prime}$ of $\zeta^{\prime}(s)$.

Below we give relations between zeros of Riemann zeta-function initialized by all derivative zeros up to $\rho_{103}^{\prime}=0.97 \ldots+i 294.03 \ldots$. Usually a zero of the derivative is related with two consecutive (and nearest) zeros of the Riemann zeta-function, however, there are exceptions. We use bold characters to indicate two not consequent zeros of $\zeta(s)$ which are related by some zero of $\zeta^{\prime}(s)$, for example $(\mathbf{2 7}, \mathbf{2 9})_{\mathbf{1 9}}$. Italic characters are used for two consequent zeros of $\zeta(s)$ if they are 'enclosed' by another pair of related zeros, for example $(27,28)_{18}$ is 'enclosed' by $(\mathbf{2 7}, \mathbf{2 9})_{19}$ (see, Figure 3 ).

$$
(2,3)_{1},(4,5)_{2},(6,7)_{3},(7,8)_{4},(9,10)_{5},(10,11)_{6},(12,13)_{7},
$$$$
(13,14)_{8},(15,16)_{9},(16,17)_{10},(17,18)_{11},(19,20)_{12},(20,21)_{13} \text {, }
$$$$
(22,23)_{14},(23,24)_{15},(24,25)_{16},(26,27)_{17},(27,28)_{18},(\mathbf{2 7}, \mathbf{2 9})_{\mathbf{1 9}} \text {, }
$$$$
(30,31)_{20},(31,32)_{21},(32,33)_{22},(34,35)_{23},(35,36)_{24},(36,37)_{25} \text {, }
$$$$
(\mathbf{3 8}, \mathbf{4 0})_{\mathbf{2 6}},(39,40)_{27},(40,41)_{28},(42,43)_{29},(43,44)_{30},(44,45)_{31} \text {, }
$$ 


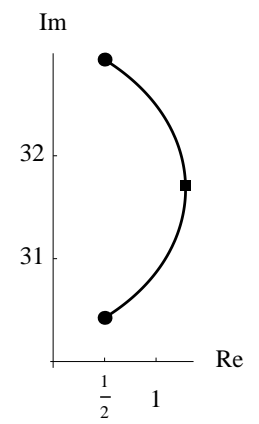

Figure 2. Parametric graphics of two solutions $s_{1}(x)$ and $s_{2}(x)$ (with parameter $x \in[0,1])$ of the equation (3.1) for $a=\zeta\left(\rho_{1}^{\prime}\right)$. The solution $s_{1}(x)$ connects the second zero $\rho_{2}=s_{1}(0)$ (lowest dot in the figure) of $\zeta(s)$ with the first zero $\rho_{1}^{\prime}=s_{1}(1)$ (small square) of $\zeta^{\prime}(s)$. The second solution $s_{2}(x)$ connects the third zero $\rho_{3}=s_{2}(0)$ (upper dot) of $\zeta(s)$ with $\rho_{1}^{\prime}=s_{1}(1)$. For the relation between $\rho_{2}, \rho_{3}$ and $\rho_{1}^{\prime}$ we use the notation $(2,3)_{1}$.

$(45,46)_{32},(47,48)_{33},(48,49)_{34},(49,50)_{35},(51,52)_{36},(52,53)_{37}$, $(53,54)_{38},(54,55)_{39},(56,57)_{40},(57,58)_{41},(\mathbf{5 7}, 59)_{42},(\mathbf{5 7}, \mathbf{6 0})_{43}$, $(61,62)_{44},(62,63)_{45},(63,64)_{46},(65,66)_{47},(66,67)_{48},(67,68)_{49}$, $(68,69)_{50},(\mathbf{7 0}, \mathbf{7 2})_{\mathbf{5 1}},(71,72)_{52},(72,73)_{53},(\mathbf{7 2 , 7 4})_{\mathbf{5 4}},(75,76)_{55}$, $(76,77)_{56},(77,78)_{57},(78,79)_{58},(80,81)_{59},(81,82)_{60},(82,83)_{61}$, $(84,87)_{62},(85,87)_{63},(86,87)_{64},(87,88)_{65},(88,89)_{66},(90,91)_{67}$, $(91,92)_{68},(\mathbf{9 1}, \mathbf{9 3})_{\mathbf{6 9}},(93,94)_{70},(\mathbf{9 5}, \mathbf{9 7})_{\mathbf{7 1}},(96,97)_{72},(97,98)_{73}$, $(98,99)_{74},(100,101)_{75},(101,102)_{76},(102,103)_{77},(103,104)_{78}$, $(104,105)_{79},(106,107)_{80},(107,108)_{81},(108,109)_{82},(\mathbf{1 0 8}, \mathbf{1 1 0})_{\mathbf{8 3}}$, $(111,112)_{84},(\mathbf{1 1 2}, \mathbf{1 1 4})_{\mathbf{8 5}},(113,114)_{86},(114,115)_{87},(\mathbf{1 1 6 , 1 1 8})_{\mathbf{8 8}}$, $(117,118)_{89},(118,119)_{90},(\mathbf{1 1 8}, \mathbf{1 2 0})_{91},(\mathbf{1 1 8}, \mathbf{1 2 1})_{\mathbf{9 2}}$,

$(\mathbf{1 2 2}, \mathbf{1 2 4})_{\mathbf{9 3}},(123,124)_{94},(124,125)_{95},(125,126)_{96},(127,128)_{97}$, $(128,129)_{98},(129,130)_{99},(130,131)_{100},(131,132)_{101},(133,134)_{102}$, $(134,135)_{103}$.

Furthermore, we consider several zeros with large imaginary part. Here we present relations between zeros of the Riemann zeta-function initialized by all derivative zeros from $\rho_{2140}^{\prime}=1.47 \ldots+i 3001.26 \ldots$ up to $\rho_{2194}^{\prime}=0.89 \ldots+$ $i 3062.32 \ldots$

$(\mathbf{2 4 7 0}, \mathbf{2 4 7 4})_{\mathbf{2 1 4 0}}, \quad(2470,2471)_{2141}, \quad(2472,2473)_{2142}$,

$(2473,2474)_{2143}, \quad(2474,2475)_{2144}, \quad(2474,2476)_{2145}$,

$(2476,2477)_{2146}, \quad(\mathbf{2 4 7 4}, \mathbf{2 4 7 8})_{\mathbf{2 1 4 7}}, \quad(2479,2480)_{2148}$,

$(2480,2481)_{2149}, \quad(2481,2482)_{2150}, \quad(\mathbf{2 4 8 1}, \mathbf{2 4 8 4})_{2151}$,

$(2483,2484)_{2152},(2484,2485)_{2153},(2485,2486)_{2154}$,

$(2486,2487)_{2155},(\mathbf{2 4 8 8}, \mathbf{2 4 9 0})_{\mathbf{2 1 5 6}},(2489,2490)_{2157}$,

$(2490,2491)_{2158},(2491,2492)_{2159},(2492,2493)_{2160}$,

$(2493,2494)_{2161},(2495,2496)_{2162},(\mathbf{2 4 9 6}, \mathbf{2 5 0 2})_{\mathbf{2 1 6 3}}$,

$(2497,2500)_{2164},(2498,2499)_{2165},(2499,2500)_{2166}$,

$(2500,2502)_{2167},(2501,2502)_{2168},(2502,2503)_{2169}$,

$(2503,2504)_{2170},(2504,2505)_{2171},(2506,2507)_{2172}$, 


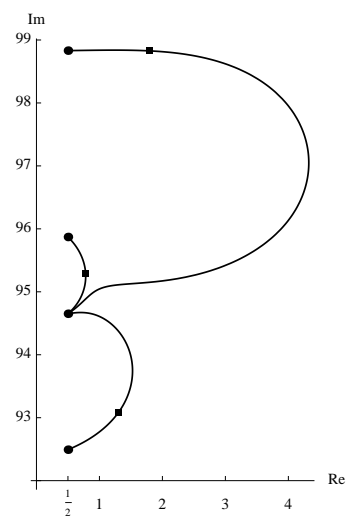

Figure 3. Graphics corresponds to the list $(26,27)_{17},(27,28)_{18},(\mathbf{2 7 , 2 9})_{19}$. Dots denote 26 th -29 th zeros of $\zeta(s)$. Small squares denote 17th, 18th and 19th zeros of $\zeta^{\prime}(s)$. We see that the consecutive zeros $\rho_{26}$ and $\rho_{27}$ are related by $\rho_{17}^{\prime}$. Nonconsecutive zeros $\rho_{27}$ and $\rho_{29}$ are related by $\rho_{19}^{\prime}$. The triple $(27,28)_{18}$ is 'enclosed' by the triple $\mathbf{( 2 7 , 2 9 )} \mathbf{1 9}$.

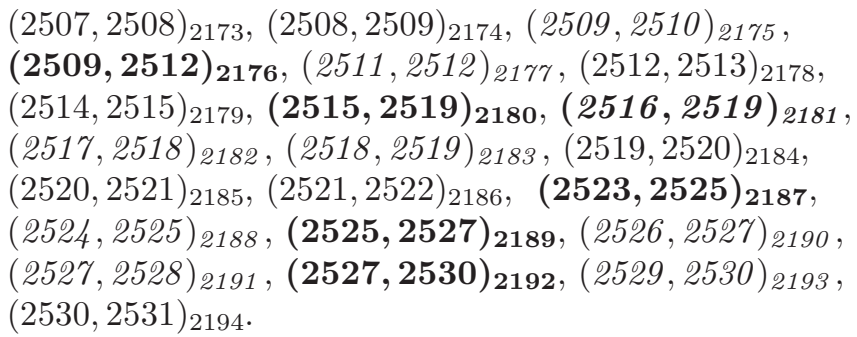

Here we find quite complicated system of related zeros, see Figure 4.

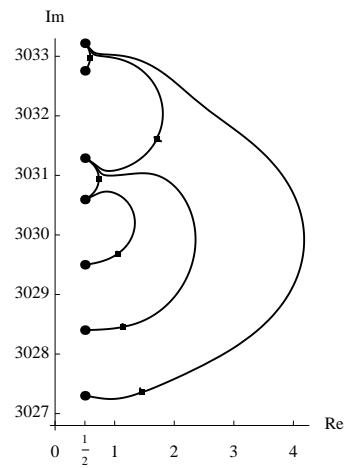

Figure 4. Graphics corresponds to the list $(\mathbf{2 4 9 6}, 2502)_{2163},(2497,2500)_{2164}$, $(2498,2499)_{2165},(2499,2500)_{2166},(2500,2502)_{2167},(2501,2502) 2168$. Dots denote $2496 \mathrm{th}-2502$ nd zeros of $\zeta(s)$. Small squares denote $2163 \mathrm{rd}-2168$ th zeros of $\zeta^{\prime}(s)$. The triple $\left(\mathbf{2 4 9 6 , 2 5 0 2 )} 2163^{\prime}\right.$ encloses' five over triples.

We note that the above connection between zeros of the Riemann zetafunction and zeros of its derivative first appeared in the proof of Speiser's 
classical equivalent for the Riemann hypothesis.

Theorem [A. Speiser]. The Riemann Hypothesis is true if and only if all zeros of the derivative $\zeta^{\prime}(s)$ in the right half-plane have real-part $\geq 1 / 2$.

Proof. Suppose that $\zeta^{\prime}\left(\rho^{\prime}\right)=0$, where $\Re\left(\rho^{\prime}\right)<1 / 2$. We have to find a zero of the function on the left of the critical line. We can assume that $\zeta\left(\rho^{\prime}\right) \neq$ 0 , because if this were the case we would have already finished. Since the derivative vanishes, there exist two opposite lines, of constant argument and along which $|\zeta(s)|$ decreases. We follow these two lines, and we must reach a zero, because $|\zeta(s)|$ decreases. If it is on the left of the critical line, we have finished, while, in the other case, we will reach the critical line. A complete proof can be found in Speiser [4] and Arias-de-Reyna [1], respectively.

If we consider the nontrivial zeros of the zeta-function as vertices and each trajectory as an edge, we obtain an infinite graph which we shall call the $\zeta$ graph. This $\zeta$-graph encodes a lot of interesting information about the zerodistribution. For instance, the above calculations suggest that this graph is not connected. The number of connected components seems to be approximately equal to the surplus of zeros of the derivative over zeta zeros, which is, up to an error of size $O(\log T)$,

$$
\frac{T}{2 \pi} \log 2=\frac{T}{2 \pi} \log \frac{T}{\pi e}-\frac{T}{2 \pi} \log \frac{T}{2 \pi e}
$$

(see [3]). We may ask whether all possible constellations appear; for example, we find all types of edges $(n, n+k)_{m}$ with $k=1, \ldots, 4$ in our data set and there is no reason why for some given value $k \geq 5$ we shall not find such an edge. Actually, the following heuristic reasoning gives a support to this statement. By Voronin's famous universality theorem (see Voronin [6], resp. $[3]$ ), the Riemann zeta-function can approximate any non-vanishing analytic function $f(s)$, defined on a compact subset $K$ of $\frac{1}{2}<\sigma<1$ with connected complement, uniformly by certain vertical shifts $\zeta(s+i \tau)$. If we construct a target-function $f$ such that its $f$-graph (defined in an analogous manner as for $\zeta)$ contains an edge we want to find for $\zeta$, then universality provides a shift with

$$
\max _{s \in K}|\zeta(s+i \tau)-f(s)|<\epsilon,
$$

and we may hope to find the edge in the $\zeta$-graph shifted by $\tau$. Of course, this is not a proof, however, it provides an approach to search for edges. Unfortunately, the universality theorem is not effective, as the only estimates for $\tau$ are astronomical. For the construction of the appropriate target-function, however, we may restrict on polynomials.

\section{Extrema and Zeros}

The periodic zeta-function is for $\sigma>1$ defined by

$$
L(s, \lambda)=\sum_{n=1}^{\infty} \frac{e^{2 \pi i \lambda n}}{n^{s}},
$$


where $\lambda \in \mathbb{R}$. For $\lambda \notin \mathbb{Z}$ this function has an analytic continuation to the whole complex plane. It is easily seen that $L(s, 1)=\zeta(s)$ and

$$
\zeta(s, 1 / 2)=\left(2^{s}-1\right) \zeta(s) .
$$

The periodic zeta-function is connected to the polylogarithm

$$
L i_{s}(z)=\sum_{n=1}^{\infty} \frac{z^{n}}{n^{s}}
$$

The last expression defines an analytic function of $z$ in the cut plane for any $s$ not a positive integer (see [5]). Then by $L(s, \lambda)=L i_{s}(\exp (2 \pi i \lambda))$ we get, for $0<\lambda<1$ and $s \notin \mathbb{N}$, that

$$
\begin{aligned}
& \frac{\partial L(s, \lambda)}{\partial \lambda}=2 \pi i L(s-1, \lambda), \\
& \frac{\partial \Re L(s, \lambda)}{\partial \lambda}=-2 \pi \Im L(s-1, \lambda), \quad \frac{\partial \Im L(s, \lambda)}{\partial \lambda}=2 \pi \Re L(s-1, \lambda) .
\end{aligned}
$$

This leads to the following statement.

Proposition 2. Let $0<\Re \rho<1$. Then $\rho$ is a nontrivial zero of the Riemann zeta-function if and only if

$$
\left.\frac{\partial \Re L(\rho+1, \lambda)}{\partial \lambda}\right|_{\lambda=\frac{1}{2}}=0,\left.\quad \frac{\partial \Im L(\rho+1, \lambda)}{\partial \lambda}\right|_{\lambda=\frac{1}{2}}=0 .
$$

We see that both functions, $R(\lambda)=\Re L(\rho+1, \lambda)$ and $I(\lambda)=\Im L(\rho+1, \lambda)$, have critical points at $\lambda=1 / 2$. We say that the nontrivial zero $\rho$ of the Riemann zeta-function has signature $(+, 0)$ if $R(\lambda)$ has a local maximum at $\lambda=1 / 2$ and $I(\lambda)$ has an inflection at $\lambda=1 / 2$. Other signatures are $(-,-),(-, 0),(-,+)$, $(0,-),(0,+),(+,-),(+,+)$.

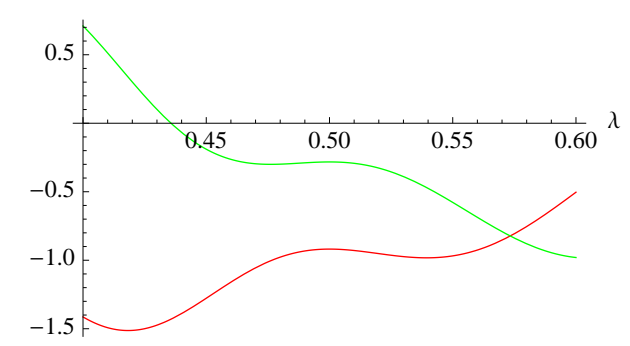

Figure 5. Graphics of real (red) and imaginary (green) parts of $L\left(\rho_{2}, \lambda\right), \lambda \in[0.4,0.6]$, where $\rho_{2}=1 / 2+21.02 \ldots$ is a second nontrivial zero of the Riemann zeta-function. Each function has a maximum at $\lambda=1 / 2$. Thus the zero $\rho_{2}$ has the signature $(+,+)$.

See Figure 5 for the signature of the second zero of the Riemann zetafunction. 
Note that the signature $(0,0)$ is impossible because

$$
\begin{aligned}
& \frac{\partial^{2} \Re L(\rho+1, \lambda)}{\partial \lambda^{2}}=-4 \pi^{2} \Re L(\rho-1, \lambda), \\
& \frac{\partial^{2} \Im L(\rho+1, \lambda)}{\partial \lambda^{2}}=-4 \pi^{2} \Im L(\rho-1, \lambda)
\end{aligned}
$$

and the Riemann zeta function has no nontrivial zeros to the left-hand side of $\sigma=0$.

Next we give signatures of the first ten nontrivial zeros of the Riemann zeta-function.

$$
(+,-),(+,+),(+,-),(+,-),(+,+),(+,+),(+,-),(+,-),(+,-),(+,+) .
$$

For calculations of signatures the second derivative test (see formulas (4.2) and (4.3)) is convenient. In the table below we consider how often each signature appears. For example, in the first thousand of nontrivial zeros $37 \%$ of zeros have signature $(+,+), 37 \%-(+,-), 13 \%-(-,+)$, and $13 \%-(-,-)$. In Table 1 percents are rounded to integers.

Table 1. Signatures of nontrivial zeros of the Riemann zeta-function in the upper half-plane.

\begin{tabular}{lcccc}
\hline Range of zeros & $(+,+)$ & $(+,-)$ & $(-,+)$ & $(-,-)$ \\
\hline $1-1000$ & $37 \%$ & $37 \%$ & $13 \%$ & $13 \%$ \\
$1001-2000$ & $35 \%$ & $36 \%$ & $14 \%$ & $15 \%$ \\
$2001-3000$ & $36 \%$ & $33 \%$ & $15 \%$ & $16 \%$ \\
$3001-4000$ & $32 \%$ & $35 \%$ & $18 \%$ & $16 \%$ \\
$4001-5000$ & $34 \%$ & $34 \%$ & $16 \%$ & $17 \%$ \\
$5001-6000$ & $34 \%$ & $33 \%$ & $17 \%$ & $17 \%$ \\
$6001-7000$ & $33 \%$ & $32 \%$ & $17 \%$ & $18 \%$ \\
$7001-8000$ & $32 \%$ & $33 \%$ & $18 \%$ & $17 \%$ \\
$8001-9000$ & $34 \%$ & $34 \%$ & $16 \%$ & $16 \%$ \\
$9001-10000$ & $31 \%$ & $34 \%$ & $19 \%$ & $17 \%$ \\
\hline
\end{tabular}

Note that up to 10000 -th zero there are no zeros with signatures $(+, 0)$, $(0,+),(0,-),(-, 0)$. We expect that each of the sign patterns appears 25 percent when sufficiently many zeros are considered; for low ranges of zeros the preference of patterns starting with ' + ' might be related to the dominance of the constant term 1 in approximations by Dirichlet polynomials.

\section{Conclusions}

There are many unanswered questions concerning the distribution of zeros of the Riemann zeta-function. One may hope that new perspectives on these problems provide new ideas to get a better understanding. We give various classifications of the zeros of the zeta-function $\zeta(s)$. Firstly, we interpret the 
zeros of $\zeta(s)$ as zeros of the Hurwitz zeta-function and, secondly, building on a relation with zeros of the derivative of $\zeta(s)$; finally, we consider the Riemann zeta-function zeros in context with the periodic zeta-function.

\section{References}

[1] J. Arias de Reyna. X-Ray of Riemann's Zeta-function. Pre-print, 2003. arXiv:math/0309433v1

[2] R. Garunkštis and J. Steuding. On the distribution of zeros of the Hurwitz zeta-function. Math. Comput., 76:323-337, 2007. Doi:10.1090/S0025-5718-06-01882-5.

[3] A. Laurinčikas and R. Garunkštis. The Lerch zeta-function. Kluwer, Dordrecht, 2002 .

[4] A. Speiser. Geometrisches zur Riemannschen Zetafunktion. Math. Ann., 110:514521, 1934. Doi:10.1007/BF01448042.

[5] C. Truesdell. On a function which occurs in the theory of the structure of polymers. Ann. Math., 46:144-157, 1945. Doi:10.2307/1969153.

[6] S.M. Voronin. Theorem on the 'universality' of the riemann zeta-function. Math. USSR Izv., 9:443-445, 1975. translated from Russian: Izv. Akad. Nauk SSSR, Ser. Matem. 39:475-486, 1975 